\title{
Consulta de Enfermagem na Atenção Primária à Saúde: Revisão Integrativa
}

\author{
Nursing Consultation in Primary Health Care: Integrative Revision
}

\author{
Stella Godoy Silva e Lima*a; Regina Stella Spagnolo ${ }^{\text {a }}$; Carmen Maria Casquel Monti Juliania; Violeta Campolina \\ Fernandes ${ }^{\text {a }}$ Lucía Silva ${ }^{\text {b; Luana Bassetto Martin }}$
}

\begin{abstract}
${ }^{a}$ Universidade Estadual Paulista, Faculdade de Medicina de Botucatu. SP, Brasil.
bUniversidade Federal de São Paulo, Escola Paulista de Enfermagem. SP, Brasil.

*E-mail: stella.godoy.lima@hotmail.com
\end{abstract}

\begin{abstract}
Resumo
Na Atenção Primária em Saúde, a consulta de enfermagem é apresentada como importante estratégia tecnológica de cuidado utilizada pelos enfermeiros, também considerada de caráter resolutivo na promoção de saúde. Este trabalho tem por objetivo analisar as evidências científicas, nacionais e internacionais, sobre a consulta de enfermagem na Atenção Primária à Saúde. Trata-se de revisão integrativa a partir das bases de dados: Lilacs, Scopus, Web of Science, Medline, IBECS e Cinahl, sendo incluídos artigos publicados no período de 2013 a 2018. Os critérios de inclusão foram artigos completos, nos idiomas português, inglês e espanhol, originados de periódicos nacionais e internacionais, excluindo artigos repetidos, do tipo revisão e comentários, bem como estudos, cujo objeto principal não se relacionava com a pergunta do estudo. Foram selecionados 14 artigos que desvelaram quatro categorias analíticas: 1) consulta de enfermagem para o fortalecimento da Atenção Primária em Saúde; 2) sistematização da assistência de enfermagem como instrumento de sustentação da consulta de enfermagem; 3) práticas da consulta de enfermagem e o uso de protocolos; e 4) desafios para implementação da consulta de enfermagem. Os enfermeiros podem utilizar estes resultados para melhoria da Consulta de Enfermagem, em todos os ciclos da vida, em um cenário de práticas dinâmicas, e em constante transformação como a Atenção Primária.
\end{abstract}

Palavras-chave: Processo de Enfermagem. Enfermagem no Consultório. Atenção Primária à Saúde.

\begin{abstract}
In primary health care, nursing consultation is presented as an important technological care strategy used by nurses, also considered to be decisive in health promotion. This work aims to analyze the scientific evidence, national and international, on nursing consultation in primary health care. This is an integrative review based on the databases: LILACS, Scopus, Web of Science, Medline, IBECS and CINAHL, including articles published from 2013 to 2018. Inclusion criteria were full articles, in Portuguese, English and Spanish, originating from national and international journals, excluding repeated articles, such as review and comments, as well as studies whose main object was not related to the study question. 14 articles were selected that unveiled four analytical categories: 1) Nursing consultation to strengthen primary health care; 2) Systematization of nursing care as an instrument to support nursing consultation; 3) Nursing consultation practices and the use of protocols; and 4) Challenges for implementing the nursing consultation. Nurses can use these results to improve the Nursing Consultation, in all life cycles in a scenario of dynamic practices and in constant transformation such as Primary Care.
\end{abstract}

Keywords: Nursing Process. Office Nursing. Primary Health Care.

\section{Introdução}

Internacionalmente, a Atenção Primária à Saúde (APS) se apresenta como estratégia de organização da atenção à saúde responsabilizando-se por uma assistência regionalizada, contínua e sistematizada para aa maior parte das necessidades da população, associando atividades de prevenção de agravos e de promoção, recuperação e reabilitação da saúde, abrangendo tanto o cuidado individual como da comunidade (BOUSQUAT et al., 2016).

No Brasil, a APS assumiu os princípios da Reforma Sanitária, e do Sistema Único de Saúde (SUS) para reorientação do modelo assistencial, a partir de um sistema universal e integrado de atenção à saúde. Em 1994, no intuito de aprimorar a cobertura assistencial nas comunidades, surgiu o Programa Saúde da Família (PSF), hoje nominada de Estratégia Saúde da Família (ESF), que adquiriu sua centralidade na APS, propondo um modelo de atenção com responsabilização e vínculo com o território de saúde (BOUSQUAT et al., 2016; ESCOREL et al., 2007; MATTA; MOROSINI, 2009).

A enfermagem, nesse contexto, contribui para o fortalecimento da APS, visto que é responsável pela atuação junto à comunidade, em um processo que parte da identificação de suas necessidades até o completo atendimento de seus pacientes, por meio da Consulta de Enfermagem (CE). Embora o cuidado direto ao paciente seja o princípio da assistência de enfermagem, o papel dos enfermeiros também perpassa a gestão e o planejamento dos cuidados.

Compete ao enfermeiro de APS tanto a gestão da unidade de saúde, por meio de ações voltadas para a melhoria da comunidade, junto a equipe multiprofissional, assim como a assistência clínica em atendimentos no consultório, ambos exigem o processo de levantamento de problemas, de 
diagnósticos, de intervenções e de avaliações.

Assim, a CE é considerada fundamental para execução de suas práticas assistenciais utilizando o Processo de Enfermagem (PE) e se constitui como um espaço de atuação privativo do enfermeiro, sendo instituída pela Lei $n^{\circ} 7.498$, de 25 de junho de 1986, na qual exige conhecimento científico, ético, estratégico, sociopolítico, de comunicação, pensamento crítico, criatividade e raciocínio clínico. Sendo uma estratégia tecnológica importante da categoria profissional, que permite o acompanhamento e a detecção precoce de agravos à saúde, favorecendo o vínculo com o paciente e a educação em saúde como uma ferramenta importante (ALVES et al.,2013; BARROS et al., 2015; DANTAS; SANTOS; TOURINHO, 2016).

Para o incentivo e o reconhecimento da enfermagem, em 2018, no Reino Unido, foi desencadeada a campanha Nursing Now, apoiada pela OMS, OPAS e o Burdett Trust for Nursing, com objetivo de melhorar a saúde global, evidenciando a prática do enfermeiro. Países como Estados Unidos, Canadá, Jamaica e Brasil estão entre os países que apoiam esta ação (CASSIANI; ROSALES, 2016). Tanto que a OMS celebra 2020 como o ano internacional de profissionais de enfermagem e obstetrícia.

No contexto da APS, a CE é apresentada como importante estratégia tecnológica de cuidado utilizada pelos enfermeiros e considerada de caráter resolutivo na promoção de saúde, porém diante de outras funções é menosprezada.

Nesse sentido, ao considerar que a CE é a principal atribuição do enfermeiro na APS e exige inúmeros conhecimentos para sua prática, a ampliação desse justifica o estudo que teve como objetivo analisar as evidências científicas, nacionais e internacionais, sobre a consulta de enfermagem na APS.

\section{Desenvolvimento}

\subsection{Método}

Trata-se de uma revisão integrativa, conforme o referencial metodológico de Ganong (1987), que permite a construção de uma análise ampla da literatura com a obtenção, a identificação, a análise e a síntese de publicações referentes a um tema específico, a partir de seus passos: definição do problema, seleção da amostra, caracterização dos estudos, análises de resultados, apresentação e discussão dos achados e apresentação da revisão (GANONG, 1987).

A coleta de dados ocorreu em novembro de 2018, por acesso online às bases de dados de saúde disponíveis nos portais Periódicos da Coordenação de Aperfeiçoamento de Pessoal de Nível Superior - Capes e Bireme - Biblioteca Virtual em Saúde (BVS). No portal CAPES, foram consultadas as seguintes bases de dados: Cumulative Index to Nursingand Allied Health Literature (Cinahl), Web of Science, US National Library of Medicine (Medline), Literatura Latinoamericana e do Caribe em Ciências da Saúde (Lilacs) e Scopus. Na busca pelo portal Bireme, Biblioteca Virtual em Saúde (BVS) foram adicionados artigos das bases de dados do Índice Bibliográfico Español de Ciencias de la Salud (IBECS), Medline e Lilacs.

Os descritores foram utilizados com os operadores booleanos OR e AND, nos idiomas português e inglês acompanhados de seus sinônimos referenciados pelos Descritores em Saúde (DeCS) / Medical Subject Headings (MeSH): Processo de Enfermagem; Enfermagem no Consultório; Atenção Primária à Saúde, Nursing Process; Office Nursing; Primary Health Care; Advanced Practice Nursing.

A partir dos artigos levantados com esta estratégia de busca, foi aplicado o filtro com relação ao período e palavras no título do artigo. Foram incluídos artigos completos relacionados ao objeto de pesquisa, nos idiomas português, inglês e espanhol, originados de periódicos nacionais e internacionais, indexados nas bases de dados referidas, no período de 2013 a novembro de 2018, em virtude de abranger artigos recentes. Foram excluídos artigos repetidos, do tipo revisão e comentários, bem como estudos, cujo objeto principal não se relacionava com a pergunta do estudo.

As seleções dos estudos foram realizadas por dois autores e a discordância entre eles foram resolvidas por um terceiro. Para o processo de seleção, os artigos foram aplicados em planilha do Excel®.

Visando sistematização dos dados, desenvolveu-se um instrumento contendo informações referentes à autoria (nome dos autores, profissão e local de atuação) e às publicações (base de dados indexados, título, ano, periódico, idioma, país de origem, natureza do estudo, objetivo de estudo, sujeitos e principais resultados).

Após a leitura do material se utilizou como estratégia metodológica qualitativa para organizar, sistematicamente, a produção do conhecimento, a técnica de análise de conteúdo, que propõe uma sequência de análise nas seguintes etapas: pré-analise, exploração do material, tratamento dos resultados, inferências e interpretação. Inicialmente foram realizadas leituras dos documentos para melhor familiarização com o texto. Em seguida se procedeu identificação de unidades de registro, contexto, núcleos de sentido e categorias. Por último, os dados foram sistematizados com posterior discussão com a literatura (BARDIN, 2011).

A discussão foi apoiada no referencial da APS e buscou alguma aproximação ao referencial teórico da Prática Avançada de Enfermagem, segundo a Ampliação do papel dos Enfermeiros na Atenção Primária à Saúde (OPAS, 2018).

Foram localizados 120 artigos nas bases; após aplicados os critérios de elegibilidade e exclusão, resultaram em um corpus de análise de 14 artigos

\subsection{Resultados}

A amostra de revisão compõe 14 artigos com foco principal na APS. Os demais estudos excluídos enfatizaram 
doenças tratadas em APS, saúde mental, pediatria, geriatria, saúde da mulher, gestante, ambientes hospitalares, oncologia, dimensionamento de enfermagem e ética profissional limitando o número da amostra.

Entre os 14 (100\%) artigos, nove (64,2\%) estão disponíveis em Língua Portuguesa e publicados em 2015 e 2016 com 4 (28,5\%) em cada ano. O Brasil foi o país que mais contribuiu com o tema com 9 (64,2\%) artigos. Considerando o primeiro autor de cada artigo, 11 atuavam como professores em Universidades (78,5\%). Quanto ao método adotado, 50\% foram de abordagem qualitativa. Em relação aos autores principais, $12(85,7 \%)$ artigos foram escritos por enfermeiros e desses, $11(78,5 \%)$ foram publicados em revistas de Enfermagem. O Quadro 1 caracteriza a amostra.

Quadro 1 - Identificação dos artigos conforme título, autores, ano de publicação, método e objetivo de estudo incluídos na revisão, 2019

\begin{tabular}{|c|c|c|c|c|c|}
\hline & Título & Ano & Método & Objetivo & Resultados \\
\hline 1 & $\begin{array}{l}\text { Ações e interações } \\
\text { na prática clínica do } \\
\text { enfermeiro na Atenção } \\
\text { Primária à Saúde. }\end{array}$ & 2018 & Qualitativo & $\begin{array}{l}\text { Compreender as ações e } \\
\text { interações suscitadas no } \\
\text { desenvolvimento da prática } \\
\text { clínica do enfermeiro na APS } \\
\text { de um município do Sul do } \\
\text { Brasil. }\end{array}$ & $\begin{array}{l}\text { Entrevistas realizadas com } 18 \text { enfermeiras. } \\
\text { Desvelou a categoria "Reconhecendo a } \\
\text { Sistematização da Assistência de Enfermagem } \\
\text { como elemento para a prática clínica } \\
\text { de enfermagem" apoia o componente" } \\
\text { ações-interações " e é composto por duas } \\
\text { subcategorias: "Realização da prática clínica } \\
\text { em enfermagem consultas ", que apresenta os } \\
\text { desafios encontrados na realização da prática; } \\
\text { e "Destacando ferramentas de gerenciamento } \\
\text { para a prática clínica baseada em evidências", } \\
\text { que opera através de estratégias em resposta ao } \\
\text { fenômeno estudado. }\end{array}$ \\
\hline 2 & $\begin{array}{l}\text { Nursing Care Plans } \\
\text { Based on NANDA, } \\
\text { Nursing Interventions } \\
\text { Classification, and } \\
\text { Nursing Outcomes } \\
\text { Classification: The } \\
\text { Investigation of } \\
\text { the Effectiveness } \\
\text { of an Educational } \\
\text { Intervention in Greece. }\end{array}$ & 2017 & Quantitativo & $\begin{array}{l}\text { Investigar a efetividade de } \\
\text { uma intervenção educativa } \\
\text { em planos de cuidados } \\
\text { domiciliares baseados na } \\
\text { NANDA, Classificação de } \\
\text { Intervenções de Enfermagem e } \\
\text { Classificação de Resultados de } \\
\text { Enfermagem para enfermeiros } \\
\text { que atuam em unidades básicas } \\
\text { de saúde na Grécia. }\end{array}$ & $\begin{array}{l}\text { A intervenção melhorou suas habilidades na } \\
\text { nomeação dos diagnósticos de enfermagem, } \\
\text { formulação adequada e individualização } \\
\text { das características definidoras, mas não as } \\
\text { aprimorou na formulação dos resultados } \\
\text { desejados. }\end{array}$ \\
\hline 3 & $\begin{array}{l}\text { Informações em saúde } \\
\text { na ótica de enfermeiras } \\
\text { da saúde da família. }\end{array}$ & 2016 & Qualitativo & $\begin{array}{l}\text { Analisar a utilidade das } \\
\text { informações em saúde como } \\
\text { ferramenta para organizar o } \\
\text { processo de trabalho a partir } \\
\text { da ótica de enfermeiras, que } \\
\text { trabalham na Saúde da família. }\end{array}$ & $\begin{array}{l}\text { Identificou-se que as enfermeiras utilizam as } \\
\text { informações para planejamento das ações no } \\
\text { dia a dia aquém do seu potencial de uso. Apesar } \\
\text { dos sujeitos reconhecerem as potencialidades } \\
\text { das informações em saúde, estas não são } \\
\text { utilizadas para o planejamento de suas ações } \\
\text { cotidianas em saúde. As capacitações voltadas } \\
\text { para o conjunto dos recursos humanos foram } \\
\text { descritas pelos profissionais como escassas, } \\
\text { desarticuladas e desintegradas. }\end{array}$ \\
\hline 4 & $\begin{array}{l}\text { Effectiveness of nursing } \\
\text { process use in primary } \\
\text { care. }\end{array}$ & 2016 & Quantitativo & $\begin{array}{l}\text { Determinar se o cuidado } \\
\text { de pacientes atribuídos a } \\
\text { enfermeiros de cuidados } \\
\text { primários que utilizam o } \\
\text { processo de enfermagem obtêm } \\
\text { melhores resultados de saúde } \\
\text { comparando com pacientes } \\
\text { cuidados por enfermeiros, } \\
\text { que não usam o processo de } \\
\text { enfermagem. }\end{array}$ & $\begin{array}{l}\text { A extensão da assistência prestada foi maior } \\
\text { nos enfermeiros que utilizaram o processo de } \\
\text { enfermagem. Os pacientes designados para } \\
\text { esses enfermeiros têm melhor controle de suas } \\
\text { doenças crônicas e incorrem em menores custos } \\
\text { com medicamentos. }\end{array}$ \\
\hline 5 & $\begin{array}{l}\text { A consulta de } \\
\text { enfermagem como } \\
\text { tecnologia do cuidado à } \\
\text { luz dos pensamentos de } \\
\text { Bacon e Galimbert. }\end{array}$ & 2016 & $\begin{array}{l}\text { Estudo } \\
\text { teórico }\end{array}$ & $\begin{array}{l}\text { Refletir sobre a CE como } \\
\text { tecnologia do cuidado na } \\
\text { atenção primaria à saúde, na } \\
\text { perspectiva filosófica. }\end{array}$ & $\begin{array}{l}\text { O pensamento filosófico oportuniza a ampliação } \\
\text { das discussões e do escopo da enfermagem, por } \\
\text { meio de uma prática sistematizada e estruturada } \\
\text { cientificamente. A consulta de enfermagem } \\
\text { é vista como um elemento essencial para a } \\
\text { melhoria da qualidade do cuidado, tornando-o } \\
\text { mais humanizado e focalizado na pessoa, na } \\
\text { família e na comunidade. }\end{array}$ \\
\hline 6 & $\begin{array}{l}\text { Terminologia de } \\
\text { enfermagem como } \\
\text { instrumento do } \\
\text { processo de trabalho do } \\
\text { enfermeiro em saúde } \\
\text { coletiva. }\end{array}$ & 2016 & Qualitativo & $\begin{array}{l}\text { Analisar o uso das } \\
\text { terminologias de enfermagem } \\
\text { como instrumento de processo } \\
\text { de enfermagem em saúde } \\
\text { coletiva. }\end{array}$ & $\begin{array}{l}\text { As situações que interferiram no uso inadequado } \\
\text { foram o desconhecimento sobre a origem e o } \\
\text { objetivo da terminologia, a falta de treinamento } \\
\text { e o uso não obrigatório. }\end{array}$ \\
\hline
\end{tabular}




\begin{tabular}{|c|c|c|c|c|c|}
\hline & Título & Ano & Método & Objetivo & Resultados \\
\hline 7 & $\begin{array}{l}\text { Sistematização } \\
\text { da assistência de } \\
\text { enfermagem na atenção } \\
\text { básica: o que dizem os } \\
\text { enfermeiros? }\end{array}$ & 2015 & Qualitativo & $\begin{array}{l}\text { Identificar o conhecimento dos } \\
\text { enfermeiros da Atenção Básica } \\
\text { em Saúde, no Brasil, sobre a } \\
\text { SAE. }\end{array}$ & $\begin{array}{l}\text { A sistematização foi associada pelos sujeitos } \\
\text { a uma sequência de passos padronizados } \\
\text { que visam gestão do cuidado; uma forma de } \\
\text { organização do serviço e ao planejamento } \\
\text { em saúde. Contudo, algo pouco presente na } \\
\text { literatura, a sistematização, por vezes foi } \\
\text { confundida como um novo modelo assistencial. }\end{array}$ \\
\hline 8 & $\begin{array}{l}\text { Percepção dos } \\
\text { enfermeiros gestores } \\
\text { da atenção primária } \\
\text { sobre processo de } \\
\text { enfermagem. }\end{array}$ & 2015 & Qualitativo & $\begin{array}{l}\text { Analisar a percepção dos } \\
\text { enfermeiros gestores da } \\
\text { atenção primária à saúde sobre } \\
\text { o processo de enfermagem. }\end{array}$ & $\begin{array}{l}\text { Os gestores reconhecem a importância } \\
\text { do processo de enfermagem, embora sua } \\
\text { implementação não seja prioridade no } \\
\text { momento. Existe dificuldade conceitual e } \\
\text { não compreensão de que a implementação da } \\
\text { metodologia de assistência deva perpassar pela } \\
\text { gestão municipal. }\end{array}$ \\
\hline 9 & $\begin{array}{l}\text { A práxis do enfermeiro } \\
\text { da estratégia de saúde } \\
\text { da família e cuidado ao } \\
\text { idoso }\end{array}$ & 2015 & Qualitativo & $\begin{array}{l}\text { Discutir a práxis do enfermeiro } \\
\text { da ESF relacionada às ações de } \\
\text { cuidado dirigidas ao idoso. }\end{array}$ & $\begin{array}{l}\text { A análise dos dados envolveu processos de } \\
\text { apreensão, de síntese, de teorização e de } \\
\text { transferência, fazendo emergir duas categorias: } \\
\text { o cuidado ao idoso e políticas públicas de saúde } \\
\text { ao idoso. Os resultados sustentam discussões } \\
\text { sobre a necessidade de aproximação entre } \\
\text { o trabalho do enfermeiro e as propostas das } \\
\text { políticas públicas de atenção à saúde do idoso. }\end{array}$ \\
\hline 10 & $\begin{array}{l}\text { A process-based } \\
\text { framework to guide } \\
\text { nurse practitioners } \\
\text { integration into primary } \\
\text { healthcare teams: } \\
\text { results from a logic } \\
\text { analysis. }\end{array}$ & 2015 & Qualitativo & $\begin{array}{l}\text { Apresentar os métodos } \\
\text { utilizados para desenvolver os } \\
\text { temas e discutir um modelo } \\
\text { integrativo de suporte de } \\
\text { integração de enfermeira } \\
\text { centrada em torno desses } \\
\text { temas. }\end{array}$ & $\begin{array}{l}\text { Cinco temas centrais que precisam ser levados } \\
\text { em consideração ao integrar enfermeiros } \\
\text { profissionais em equipes de atenção primária } \\
\text { foram identificados. Esses temas são: } \\
\text { planejamento, definição de função, modelo de } \\
\text { prática, colaboração e suporte à equipe. }\end{array}$ \\
\hline 11 & $\begin{array}{l}\text { Caracterização das } \\
\text { dissertações de teses } \\
\text { brasileiras acerca da } \\
\text { interface processo de } \\
\text { enfermagem e atenção } \\
\text { primária }\end{array}$ & 2014 & Quantitativo & $\begin{array}{l}\text { Caracterizar as dissertações } \\
\text { e teses disponíveis no banco } \\
\text { de teses da CAPES que } \\
\text { versem sobre o processo } \\
\text { de enfermagem na atenção } \\
\text { primária. }\end{array}$ & $\begin{array}{l}\text { Visualizou-se uma incipiência de produções } \\
\text { científicas, evidenciando-se a necessidade } \\
\text { de desenvolvimento de novas investigações. } \\
\text { Experiências positivas do uso do processo } \\
\text { de enfermagem na atenção primária foram } \\
\text { elucidadas, contribuindo para afirmar seus } \\
\text { benefícios em tal nível de complexidade e a } \\
\text { essencialidade dessa interface. }\end{array}$ \\
\hline 12 & $\begin{array}{l}\text { Positive practice } \\
\text { environments influence } \\
\text { job satisfaction of } \\
\text { primary health care } \\
\text { clinic nursing managers } \\
\text { in two South African } \\
\text { provinces }\end{array}$ & 2014 & Quantitativo & $\begin{array}{l}\text { Determinar a satisfação geral } \\
\text { do trabalho da clínica de APS } \\
\text { de gestores de enfermagem } \\
\text { e os preceptores de sua } \\
\text { satisfação no trabalho em duas } \\
\text { províncias sul-africanas. }\end{array}$ & $\begin{array}{l}\text { Permitir que os enfermeiros tenham maior } \\
\text { escolha de clínica para trabalhar, a prevenção } \\
\text { da violência e o endereçamento de cargas de } \\
\text { trabalho podem melhorar o ambiente de prática } \\
\text { e a satisfação no trabalho dos gerentes de } \\
\text { enfermagem da APS }\end{array}$ \\
\hline 13 & $\begin{array}{l}\text { Vivenciando a } \\
\text { classificação } \\
\text { internacional de } \\
\text { práticas de enfermagem } \\
\text { em saúde coletiva: } \\
\text { relato de experiência. }\end{array}$ & 2013 & Quantitativo & $\begin{array}{l}\text { Realizar relato de experiência } \\
\text { acerca da construção de } \\
\text { roteiros para CE utilizando } \\
\text { diagnostico de enfermagem na } \\
\text { perspectiva CIPESC articulada } \\
\text { a SAE. }\end{array}$ & $\begin{array}{l}\text { Os encontros visaram ao aprofundamento } \\
\text { teórico acerca da temática e à construção dos } \\
\text { roteiros para nortear as consultas realizadas } \\
\text { pelos educandos. } \\
\text { Constataram-se resultados positivos, os quais } \\
\text { provam a viabilidade da utilização deste } \\
\text { instrumento, e negativos, o que indica a } \\
\text { deficiência da formação dos profissionais na } \\
\text { perspectiva da sistematização da assistência no } \\
\text { contexto da Atenção Primária à Saúde. }\end{array}$ \\
\hline 14 & $\begin{array}{l}\text { Variable que } \\
\text { condicionan la } \\
\text { utilización de la } \\
\text { consulta de enfermeira } \\
\text { em centros de salud } \\
\text { de la comunidad de } \\
\text { Madrid }\end{array}$ & 2013 & Quantitativo & $\begin{array}{l}\text { Reconhecer quais fatores } \\
\text { influentes ao uso do cuidado de } \\
\text { enfermagem na atenção básica. }\end{array}$ & $\begin{array}{l}\text { As diferenças no uso da consulta de enfermagem } \\
\text { respondem a critérios de necessidade, mas } \\
\text { também são influenciadas pelas condições de } \\
\text { acessibilidade. }\end{array}$ \\
\hline
\end{tabular}

Fonte: Dados da pesquisa.

Após leitura do corpus de análise foram sistematizados, qualitativamente, os dados segundo análise de conteúdo emergindo quatro categorias analíticas relacionadas à consulta de enfermagem na APS: 1) consulta de enfermagem para o fortalecimento da atenção primária em saúde; 2) sistematização da Assistência de Enfermagem como instrumento de sustentação da consulta de enfermagem; 3) práticas da consulta de enfermagem e o uso de protocolos; e 4) desafios para implementação da consulta de enfermagem. 


\subsection{Discussão}

\subsubsection{Consulta de enfermagem para o fortalecimento da atenção primária em saúde}

Esta categoria demonstra que a CE é um diferencial dentro da APS e deve ser valorizada. Revelou que a CE tem como estratégia eficaz identificar, de forma precoce, desvios no processo de adoecimento e, também, acompanhamento a todos os grupos populacionais.

A Organização Pan Americana de Saúde (OPAS) junto à Organização Mundial da Saúde (OMS) tem incentivado a formação do Enfermeiro de Prática Avançada (EPA), definida pelo Conselho Internacional de Enfermagem como enfermeiro registrado, com base de conhecimentos especializados, habilidades complexas de tomada de decisões e competências clínicas para prática expandida, cujas características são moldadas pelo contexto e/ou país em que está credenciado (INTERNACIONAL COUNCIL OF NURSE PRACTITIONER AND ADVANCED PRACTICE NURSIG, 2013).

As práticas avançadas foram implementadas a princípio em países como Canadá e Estados Unidos, classificando-as como uma formação profissional de nível avançado. Este avanço tem sido reconhecido pelos órgãos políticos e pacientes como planejamento eficiente de qualidade garantindo a integração da prática multiprofissional da APS, com o gerenciamento e o cuidado de indivíduos com doenças agudas leves e crônicas, sob a orientação de protocolos clínicos ou diretrizes (MIRANDA NETO et al., 2018).

Assim, a prática avançada está sendo considerada uma alternativa eficaz e eficiente para APS, nos serviços de saúde, em vários países, estimulando mudanças políticas com ênfase no progresso e valorização da formação do profissional de enfermagem (MUNYEWENDE; RISPEL; CHIRWA, 2014; INTERNACIONAL COUNCIL OF NURSE PRACTITIONER AND ADVANCED PRACTICE NURSIG, 2013).

No Brasil, o enfermeiro como membro da equipe multiprofissional da APS possui atribuições gerenciais, assistenciais e educativas, tendo a CE como atividade principal para dar respostas à complexidade das necessidades de saúde de indivíduos, famílias e comunidades (CASTRO et al., 2016; GASPARINO; SIMONETTI; TONETE, 2013; GRANEROMOYA et al., 2016; KAHL et al., 2018; KRAUZER et al., 2015), sendo esta alicerçada por protocolos que exercem a função de respaldar o profissional, por meio de práticas baseadas em evidências (CFE, 1986; DANTAS; SANTOS; TOURINHO, 2016; PATIRAKI et al., 2017; PIMENTA et al., 2015).

A CE tem como base os princípios de universalidade, de equidade e de integralidade das ações de saúde para melhorar a qualidade da assistência perante a sociedade (CAVALCANTE et al., 2016; SILVA; SANTOS, 2015). Dessa maneira, a
CE se torna um diferencial relevante que corrobora com os princípios instituídos pelo SUS, mesmo porque os enfermeiros assumem um papel muito importante dentro da APS, sendo estes responsáveis pelo acesso do usuário, como porta entrada na rede de atenção à saúde.

É reconhecida a importância da CE por meio dos resultados e ações assistenciais, a qual abrange o maior número de pacientes pelo fácil acesso da população e vínculo proporcionado junto ao enfermeiro (DANTAS; SANTOS; TOURINHO, 2016; MARTINS; SILVA; MARQUES, 2016; MARTIN-FERNANDEZ et al., 2013).

A expansão da CE nas Unidades Básicas de Saúde (UBS) brasileiras e, especialmente, na Estratégia Saúde da Família (ESF) promoveu a melhoria no perfil epidemiológico e de saúde, a exemplo da redução da mortalidade infantil no Brasil, com redução de 67,6\%, entre 1990 e 2015, segundo França et al. (2017). Com a consolidação da CE na APS, o enfermeiro desempenha atividades de forma contínua, com práticas clínicas centradas no ciclo vital, na assistência à família e comunidade (GASPARINO; SIMONETTI; TONETE, 2013; KRAUZER et al., 2015; SILVA; SANTOS, 2015).

Em alguns países da América Latina e Caribe, assim como no Brasil, os enfermeiros de APS, por meio da CE, possuem autonomia, autoridade legal e reguladora em parceria com o médico, para avaliar, diagnosticar e tratar pacientes com doenças agudas, lesões comuns, manejo de doenças crônicas, assim como solicitar e interpretar testes de diagnóstico, prescrever medicamentos (com base em protocolos) e executar procedimentos específicos (CONTANDRIOPOULOS et al., 2015; KAHL et al., 2018; PATIRAKI et al., 2017).

$\mathrm{Na}$ CE, o profissional tem como objetivo principal prestar assistência integral, resolutiva, contínua, com qualidade, oferecendo atendimento para as demandas da população, que utiliza os serviços de saúde, como gestantes, atendimentos de puericultura, sendo capaz de identificar não só as necessidades consideradas como demandas específicas, mas também as situações eventuais apresentadas no cotidiano e nas emergências (BARROS et al., 2015; CFE, 1986; NICHIATA et al., 2012) .

Desse modo, o enfermeiro conduz as orientações sem impor suas concepções, respeitando as crenças, as normas, os valores e a realidade socioeconômica do paciente, gerando melhor interação pessoal, que favoreça aproximação com o viver das pessoas, identificando os reais problemas de saúde para que possa intervir (DANTAS; SANTOS; TOURINHO, 2016).

Nesse sentido, a prática avançada contribui para a melhoria da qualidade da assistência, com o aumento da cobertura e a diminuição dos custos de saúde. Sua implementação é vista como importante estratégia para a consolidação da força de trabalho do profissional de enfermagem, particularmente, no que diz respeito à qualificação, ao recrutamento, ao reconhecimento e à fixação nos serviços de saúde (OPAS, 
2018).

\subsubsection{A sistematização da assistência de enfermagem como instrumento de sustentação da $\mathrm{CE}$}

O PE ao promover melhor acompanhamento do usuário, na prática assistencial, é capaz de fornecer maior cobertura para os serviços com ações de promoção e de prevenção da saúde, que contribui para a elaboração de um sistema classificatório que estimula mudanças, aprimora a qualidade da gestão, do ensino, da pesquisa e de avaliação do cuidado à saúde (KRAUZER et al., 2015; PÉREZ RIVAS et al., 2016; SILVA et al., 2017). A Sistematização da Assistência de Enfermagem (SAE), entendida como instrumento metodológico, que orienta o cuidado de enfermagem e documenta a prática da $\mathrm{CE}$ está associada ao PE, sendo extremamente importante para a organização e gerência do cuidado (ALVES et al., 2013; KRAUZER et al., 2015). Além disso, tornou-se prevista desde a década de 1970, no serviço profissional de enfermagem em todas as áreas e níveis de assistência (CONSELHO FEDERAL DE ENFERMAGEM, 1986).

A possibilidade de aplicar a SAE no cotidiano dos diferentes ambientes assistenciais torna o profissional capaz de exercer as suas funções privativas como, por exemplo, consulta, diagnóstico e prescrição de enfermagem, uma vez que não podem ser delegadas aos demais integrantes da equipe (DANTAS; SANTOS; TOURINHO, 2016; KRAUZER et al., 2015; PÉREZ RIVAS et al., 2016). Vale ressaltar que a SAE enfatiza o direcionamento da organização do cuidado e possibilita aos profissionais de enfermagem exercer sua autonomia perante os demais trabalhadores da saúde.

Existe o reconhecimento da importância da SAE e do PE para organização das atividades assistenciais e gerenciais relacionadas à equipe de enfermagem na APS, bem como de seu auxílio na tomada de decisão (DINIZ et al., 2015). Verificase que fragilidades na implementação da SAE na APS ocorre por sobrecarga de trabalho, aumento da demanda e atenções voltadas aos serviços burocráticos, os quais são exigidos pelos gestores (DINIZ et al., 2015; KAHL et al., 2018).

Uma das demandas, que se apresenta no cotidiano do enfermeiro, envolve as atividades de gestão. Além da parcela gerencial do próprio planejamento do cuidado, em muitas unidades, os profissionais acumulam o papel assistencial e o gerencial (este relacionado não apenas com a assistência de enfermagem, mas a gerência da unidade).

Conforme aponta Ferreira et al. (2018), há na APS sobrecarga de trabalho com atividades administrativas ao Enfermeiro, sejam gerenciais e de apoio ao funcionamento da unidade, a organização da demanda e infraestrutura um dilema da prática estar entre o papel "assistencial X gerencial".

Fato este que se alinha às práticas avançada por permitir que o profissional tenha alto grau de liberdade e de competência para tomar decisões clínicas, realizar avaliações, diagnósticos e prescrições, além de ser responsável pela gestão de casos, de avaliação, de implementação de programas e de planos de cuidado ao paciente (MIRANDA NETO et al., 2018; OPAS, 2018).

Dessa forma, em meio à literatura, encontram-se outras nomenclaturas, que se referem à sistematização da assistência como: processo do cuidado, metodologia do cuidado, processo de assistir, consulta de enfermagem e, inclusive, o próprio PE (DANTAS; SANTOS; TOURINHO, 2016; DINIZ et al., 2015; KRAUZER et al., 2015; PÉREZ RIVAS et al., 2016).

Evidencia-se que o uso de linguagens padronizadas é necessário para o exercício da profissão, ou seja, estimular o uso de terminologias e taxonomias universais com desfecho de saúde para demonstrar a eficácia das intervenções (PATIRAKI et al., 2017; PÉREZ RIVAS et al., 2016).

O Conselho Internacional de Enfermeiros (CIE) busca a universalização da linguagem profissional visando agilidade e prontidão na definição de diagnósticos e de intervenções de enfermagem e possibilidades de diálogo, no âmbito internacional, em distintos contextos culturais, sociais e de saúde (CAVALCANTE et al., 2016; DINIZ et al., 2015; NICHIATA et al., 2012).

Assim, desde 1919, modelos classificatórios são utilizados como instrumentos para auxiliar os profissionais no exercício de suas práticas, sendo que um dos mais relevantes é o de classificação de diagnósticos de enfermagem, coordenado desde 2002 pelo Grupo Norte-Americano de Classificação de Diagnósticos de Enfermagem, que passou a ser chamado North American Nursing Diagnoses Association - NANDA Internacional, direcionados às instituições hospitalares (ALVES et al., 2013; KRAUZER et al., 2015).

Nesse sentido, a OMS sugeriu para CIE um projeto internacional voltado para classificações realizadas na APS, chamado de Classificação Internacional para Prática de Enfermagem $\left(\mathrm{CIPE}^{\circledR}\right)$ (ALVES et al., 2013; SILVA et al., 2017).

A CIPE $^{\circledR}$ corresponde a uma terminologia multiaxial combinatória, que possibilita formular diagnósticos, intervenções e resultados de enfermagem, padronizando as terminologias, na qual representa o domínio da prática e unifica a linguagem da enfermagem em vertente mundial (ALVES et al., 2013).

No Brasil, a partir de 1994, a Associação Brasileira de Enfermagem (ABEn) assumiu o compromisso de desenvolver um projeto voltado para a consolidação da aplicação da CIPE ${ }^{\circledR}$ na Atenção Básica, originando a Classificação Internacional de Práticas de Enfermagem em Saúde Coletiva $\left(\right.$ CIPESC $\left.^{\circledR}\right)$. Tal iniciativa acarretou a criação do inventário vocabular de enfermagem em saúde coletiva, por meio da identificação de fenômenos, de ações de enfermagem e caracterização do processo de trabalho, inicialmente, na cidade de CuritibaParaná e, posteriormente, nos Estados do Rio Grande do Sul, Santa Catarina e Paraíba (ALVES et al., 2013; NICHIATA et al., 2012).

A CIPE $^{\circledR} /$ CIPESC $^{\circledR}$ é capaz de permitir a realização da evolução de enfermagem, com propósito de auxiliar nas 
intervenções e orientações fornecidas aos pacientes, sendo consideradas pelos enfermeiros da APS como uma forma prática e ágil de desempenhar suas atividades, quando integrado ao sistema informatizado na $\mathrm{CE}$, na nomeação dos diagnósticos e possibilidades nas intervenções de enfermagem (CAVALCANTE et al., 2016; SALVADOR; SANTOS; DANTAS, 2014; SILVA et al., 2017).

Sendo assim, as utilizações da SAE, bem como dos seus instrumentos de apoio são importantes para o fortalecimento da CE, pois permitem a organização do processo de trabalho e linguagem unificada. Aliado a isso, destaca-se a implementação da prática avançada como uma possibilidade de mudança na força de trabalho em saúde, principalmente, no que diz respeito às competências e às qualificações de enfermeiros nos serviços de saúde.

\subsubsection{Práticas da consulta de enfermagem e o uso de protocolos}

O uso de protocolos assistenciais tem contribuído para a realização da $\mathrm{CE}$ e para a efetivação da integralidade do cuidado proposta pelo SUS. No entanto, para que tenham significado no cotidiano do trabalho dos profissionais de saúde, esses devem ser discutidos, problematizados e adequados às necessidades da população e ao processo de trabalho das equipes (SALVADOR; SANTOS; DANTAS, 2014; SILVA et al., 2017; SILVA; SANTOS, 2015).

Utilizam-se os protocolos, principalmente, por meio da descrição de situações específicas relacionadas à assistência e ao cuidado, conduzidas pelos profissionais para a prevenção, a recuperação e a reabilitação da saúde. Entre seus atributos, estão ações de avaliação, de diagnóstico e de tratamento mediante o uso de intervenções educacionais, emocionais, físicas e farmacológicas, que a enfermagem desempenha de maneira independente ou compartilhada com outros profissionais da equipe de saúde (PATIRAKI et al., 2017; PIMENTA et al., 2015).

Ademais, a OMS sugere a elaboração de guias clínicos ou protocolos com base em evidências para atenção em saúde, na APS, que envolvam competências de agente de mudança e liderança, competências administrativas, como o desenvolvimento e implementação de políticas e tomada de decisões, e práticas avançadas que gerem inovações de maneira proativa (OPAS, 2018).

Esse conhecimento pode estar embasado no desenvolvimento das teorias de enfermagem, inicialmente, apresentadas nos Estados Unidos, que atingiram as escolas de enfermagem do Brasil, na década de 1970, colaborando com estudos, que foram realizados pelas enfermeiras brasileiras (DANTAS; SANTOS; TOURINHO, 2016; KRAUZER et al., 2015).

Nesse sentido, as Universidades brasileiras têm destacado a importância de se colocar nos currículos de graduação conteúdos que estimulem o aprendizado, a compreensão dos métodos para a realização da prática embasada em conhecimento científico (DINIZ et al., 2015; MACHADO, 2013; SILVA; SANTOS, 2015).

A busca pela construção de conhecimento específico deve ser estimulada em todos os âmbitos de trabalho da enfermagem. Aprimorar-se é uma característica da profissão para qualidade do trabalho assistencial e colaboração da ciência (ALVES et al., 2013; PATIRAKI et al., 2017).

Porém, a literatura apontou que há uma tendência na reprodução do modelo biomédico, com ações centradas nas queixas agudas, com uma proposta curativa, focada na doença e não no ser humano (KAHL et al., 2018; SILVA; SANTOS, 2015).

Entre os motivos para reprodução desse modelo se encontram fatores pessoais dos profissionais, visão cartesiana de Mundo, preparo inadequado na graduação, falta de comprometimento, envolvimento e responsabilidade, além de déficit para desempenhar liderança e organização (ALVES et al., 2013; KRAUZER et al., 2015).

Há que se estimular transformações das práticas assistenciais e incentivar ao uso de protocolos para o aprimoramento e ampliação da qualidade dos serviços. Nesse sentido, deve-se estimular o aperfeiçoamento dos profissionais, por meio de especializações, pós-graduações e educação permanente em saúde com intuito de promover reconhecimento, atualização e assistência com qualidade, respondendo às necessidades da população (KRAUZER et al., 2015; MUNYEWENDE; RISPEL; CHIRWA, 2014; PÉREZ RIVAS et al., 2016).

A formação de profissionais autônomos e qualificados pode ser estimulada por meio da realização do mestrado em práticas avançadas, que visa assegurar a qualidade dos serviços; promover a formação contínua; garantir assistência à saúde em áreas de difícil acesso e oferecer a população melhoria na competência dos profissionais (ORGANIZATION PAN AMERICAN HEALTH, 2018; INTERNACIONAL COUNCIL OF NURSE PRACTITIONER AND ADVANCED PRACTICE NURSIG, 2013).

Além disso, atividades de educação permanente devem ser desenvolvidas de forma eficaz, com a elaboração de estratégias que amenizem os problemas existentes na atualidade, incentivem a aquisição de conhecimentos e busquem aplicação dos protocolos corretamente, visto que os materiais disponíveis na rede pública, como os manuais, não são suficientes para a garantia do planejamento e gestão de acordo com as necessidades apresentadas no cotidiano (MARTINS; SILVA; MARQUES, 2016; PATIRAKI et al., 2017; SILVA; SANTOS, 2015).

Ressalta-se que as aplicações dos protocolos auxiliam e respaldam o profissional na realização da CE. No entanto, necessita-se da atualização dos instrumentos com o incentivo de adquirir novos conhecimentos e tecnologias, embasados em evidências científicas, que ampliem o cuidado de enfermagem e considerando os contextos locais. 


\subsubsection{Desafios para implementação da CE}

$\mathrm{Na}$ atualidade, a busca pela assistência em saúde com qualidade e segurança está presente, de forma primordial, nas discussões políticas e constitui um grande desafio para a sociedade (MIRANDA NETO et al., 2018; OPAS, 2018). Nesse contexto se encontram nas instituições de saúde inúmeras dificuldades relacionadas aos recursos físicos, materiais e humanos.

Alguns entraves são apontados pelos profissionais enfermeiros, como por exemplo, espaços físicos nos quais exercem suas atividades adaptados em casas residenciais, consultórios compartilhados e outras estruturas inadequadas. Envolvem também questões relacionadas aos equipamentos obsoletos, ausência de conexão à internet e problemas no sistema informatizado, que geram transtornos no dia a dia dos trabalhadores em APS (MARTINS; SILVA; MARQUES, 2016).

Quanto aos recursos humanos, especificamente, no que diz respeito aos enfermeiros, existe alta rotatividade desses profissionais na APS em função de diversas atribuições exigidas e baixa remuneração comparada às responsabilidades (ALVES et al., 2013; MARTINS; SILVA; MARQUES, 2016).

É questionada a dificuldade em exercer atividades assistenciais com interferências dos membros da equipe, principalmente, no momento da $\mathrm{CE}$, para resolução de problemas relacionados à gestão, refletindo falta de planejamento para desempenhar as atividades e trazendo como consequência a diminuição de qualidade do atendimento ao paciente (ALVES et al., 2013; SALVADOR; SANTOS; DANTAS, 2014).

Diante da sobrecarga de trabalho expressa há o descumprimento das etapas da sistematização, na CE, sendo esse fato considerado um desafio para os enfermeiros, principalmente, no quesito diagnóstico, caracterizado pela falta de habilidade e manejo com o instrumento classificatório (CAVALCANTE et al., 2016; DINIZ et al., 2015; KAHL et al., 2018; MARTINS; SILVA; MARQUES, 2016; PATIRAKI et al., 2017).

Evidenciam-se três fatores principais que interferem na concretização da SAE na CE: a princípio, os fatores pessoais e profissionais, que estão relacionados ao preparo inadequado na graduação, à falta de engajamento, responsabilidade, falta de influência e organização, problemas em relacionamentos interpessoais, desvio de funções e baixa remuneração. Os fatores organizacionais se referem à ausência de profissionais de enfermagem, às funções administrativas junto à assistência, ao desinteresse da gestão, às estruturas físicas inadequadas e ao déficit dos serviços de apoio. Por fim, os fatores pertinentes ao modelo teórico, que evidenciam a ausência da realização de todas as etapas e a complexidade do PE (SALVADOR; SANTOS; DANTAS, 2014).

São apontadas controversas conceituais, que podem dificultar a compreensão dos profissionais e estudantes durante o período de formação, pois a mesma metodologia pode ser encontrada sob diversas nomenclaturas, como: SAE, $\mathrm{CE}$, metodologia do cuidado de enfermagem, metodologia da assistência de enfermagem, planejamento da assistência de enfermagem, processo de atenção em enfermagem, processo de cuidar em enfermagem, entre outras (KRAUZER et al., 2015).

Necessita-se estimular órgãos políticos e administrativos para apoiar mudanças no perfil assistencial da enfermagem, com estratégias de respaldo a estes profissionais, promovendo maior envolvimento na prática do PE e sistematização efetiva da $\mathrm{CE}$, uma vez que a complexidade do trabalho leva à redução do número de pacientes a serem contemplados na assistência (CONTANDRIOPOULOS et al., 2015; DINIZ et al., 2015; KRAUZER et al., 2015).

Ademais, considera-se também como desafio a fragilidade dos instrumentos disponíveis, com questionamentos referentes à limitação de diagnósticos e intervenções que dão maior ênfase nas áreas de saúde da mulher e da criança evidenciando necessidade da inclusão de termos relacionados às outras áreas de cuidado (CAVALCANTE et al., 2016; SALVADOR; SANTOS; DANTAS, 2014; SILVA et al., 2017).

Neste contexto, observa-se o desconhecimento sobre tais instrumentos, falta de treinamento, não obrigatoriedade de seu uso, bem como condições de trabalho desfavoráveis, o que gera negligência da aplicação do inventário vocabular como instrumento do processo de trabalho (CAVALCANTE et al., 2016; DINIZ et al., 2015; SALVADOR; SANTOS; DANTAS, 2014).

Segundo a OMS, alguns passos são necessários para enfrentar os desafios da enfermagem: a) implementar estratégias ativas para recrutar enfermeiros com intuito de alcançar profissionais críticos, minimizando o déficit de recursos humanos em saúde; b) padronizar as exigências e as qualificações para cursos de formação, para garantir a flexibilidade profissional; c) incentivar profissionais críticos para o ensino gerando futuros pesquisadores e líderes; d) promover integração interprofissional nas equipes de saúde; e) desenvolver avanços para a prática, com trajetórias profissionais estabelecidas no início da carreira profissional (ORGANIZATION PAN AMERICAN HEALTH, 2018; INTERNACIONAL COUNCIL OF NURSE PRACTITIONER AND ADVANCED PRACTICE NURSIG, 2013).

É relevante que os enfermeiros, atuantes na APS, estejam inteirados, preparados para o exercício da CE, visando melhoria contínua da qualidade da assistência, reconhecimento profissional e planejamento do trabalho, o que requer constante atualização, por meio da educação permanente, treinamento e especializações.

\section{Conclusão}

As evidências identificam que a CE incentiva a autonomia do enfermeiro e o vínculo com o paciente, a SAE 
assegura a contemplação das etapas da consulta e incentiva a padronização universal do diagnóstico de enfermagem e os protocolos devem estar atualizados para ampliação da assistência.

A CE contribui para a qualidade da atuação do enfermeiro, pois proporciona autonomia profissional, incentivo ao planejamento e organização do trabalho visando o estabelecimento de uma prática completa e científica, amparada por políticas públicas recentes da APS.

Esta revisão aponta o trabalho do enfermeiro como agente fortalecedor da APS, por meio da consulta garantindo qualidade da assistência a todos os grupos populacionais, com ações de cuidados eficientes e aceitação da comunidade. Assim, a importância do papel do enfermeiro, no contexto atual, requer a valorização profissional e, em função da complexidade desse papel, a qualificação permanente para a melhoria contínua da atenção à saúde.

Como limitação do estudo, destaca-se o reduzido número de artigos encontrados sobre a temática com ênfase no processo de trabalho do enfermeiro, por meio da CE na APS.

Os enfermeiros, apesar dos desafios apontados, realizando a sistematização da assistência de enfermagem, com utilização de protocolos, podem utilizar estes resultados para aperfeiçoamento da $\mathrm{CE}$, em todos os ciclos da vida, em um cenário de práticas dinâmico e em constante transformação, como o da Atenção Primária, o que representa um diferencial positivo para qualquer sistema de saúde.

\section{Referências}

ALVES, K.Y.A. et al . Vivenciando a classificação internacional de práticas de enfermagem em saúde coletiva: relato de experiência. Esc. Anna Nery Rev. Enferm., v.17, n.2, p.381-388, 2013. doi: 10.1590/S1414-81452013000200025.

BARDIN, L. Análise de conteúdo. Lisboa: Edições 70, 2009.

BARROS, A.L.B.L.B. et al. Processo de enfermagem: guia para a prática. São Paulo: Coren-SP, 2015.

BOUSQUAT, A. et al. Atenção primária à saúde e coordenação do cuidado nas regiões de saúde: perspectiva de gestores e usuários. Ciênc. Saúde Coletiva, v.22, n.4, p.1141-1154, 2017. doi: 10.1590/1413-81232017224.28632016

CASSIANI, S.H.D.B.; ROSALES, L.K. Initiatives towards advanced practice nursing implementation in the region of the Americas. Esc. Anna Nery Rev. Enferm., v.20, n.4, p.4-5, 2016. doi:10.5935/1414-8145.20160081

CASTRO, M.C.F. et al. Subconjunto terminológico CIPE ${ }^{\circledR}$ com feridas tumorais malignas. Acta Paul. Enferm., v.29, n.3, p.340346, 2016. doi: 10.1590/1982-0194201600047

CAVALCANTE, M.D.M.A. et al. Terminologia de enfermagem como instrumento do processo de trabalho do enfermeiro em saúde coletiva. Rev. Esc. Enferm. USP, v.50, n.4, p.610-616, 2016. doi:10.1590/S0080-623420160000500010

CFE - Conselho Federal de Enfermagem. Lei $n^{\circ} 7.498 / 86$, de 25 de junho de 1986. Dispõe sobre a regulamentação do exercício de enfermagem e dá outras providências. Brasília: Cofen, 1986.

CONTANDRIOPOULOS, D. et al. A process-based framework to guide nurse practitioners integration into primary healthcare teams: results from a logic analysis. BMC Health Serv. Res., v.15, n.1, p.1-11, 2015. doi: 10.1186/s12913-015-0731-5

DANTAS, C.N.; SANTOS, V.E.P.; TOURINHO, F.S.V. A consulta de enfermagem como tecnologia do cuidado à luz dos pensamentos de bacon e galimberti. Texto Contexto Enferm., v.25, n.1, p.1-8, 2016. doi:10.1590/0104-0707201500002800014.

DINIZ, I.A. et al. Percepção dos enfermeiros gestores da atenção primária sobre o processo de enfermagem. Rev. Bras. Enferm., v.68, n.2, p.206-213, 2015. doi:10.1590/0034-7167.2015680204i

ESCOREL, S. et al. O Programa de Saúde da Família e a construção de um novo modelo para a atenção básica no Brasil. Rev. Panam. Salud Pública, v.21, n.2, p.164-176, 2007.

FERREIRA, S.R.S.; PÉRICO, L.A.D.; DIAS, V.R.G.F. The complexity of the work of nurses in Primary Health Care. Rev. Bras. Enferm., v.71, p.704-709, 2018. doi: 10.1590/0034-71672017-0471

FRANÇA, E.B. et al. Principais causas da mortalidade na infância no Brasil, em 1990 e 2015: estimativas do estudo de Carga Global de Doença. Rev. Bras. Epidemiol., v.20, p.46-60, 2017. doi: 10.1590/1980-5497201700050005

GASPARINO, R.F.; SIMONETTI, J.P.; TONETE, V.L.P. Consulta de enfermagem pediátrica na perspectiva de enfermeiros da Estratégia Saúde da Família. Rev. Rene., v.14, n.6, p.11121122, 2013.

GRANERO-MOYA, N. et al. Dificultades de las enfermeras de atención primaria en los procesos de planificación anticipada de las decisiones: un estudio cualitativo. Aten. Primaria, v.48, n.10, p.649-656, 2016. doi: 10.1016/j.aprim.2016.01.008

INTERNATIONAL COUNCIL OF NURSE PRACTITIONER AND ADVANCED PRACTICE NURSIG. Definition and characteristics of the role. Genebra: Internacional Council of Nurse, 2013. Disponível em: https://internacional,aanp.org/ Practice/APNRoles. Acesso em: 03 jan. 2019.

KAHL, C. et al. Ações e interações na prática clínica do enfermeiro na Atenção Primária à Saúde. Rev. Esc. Enferm. USP, v.52, p.e03327, 2018. doi: 10.1590/s1980-220x2017025503327.

KRAUZER, I.M. et al. Nursing care systematization in primary care: what do the nurses say? Cienc. Enferm., v.21, n.2, p.31-38, 2015. doi: 10.4067/S0717-95532015000200004

MACHADO, M.L.P. Consulta de enfermagem ampliada : possibilidades de formação para a prática da integralidade em saúde. Rev. Gaúch. Enferm., v.34, n.4, p.53-60, 2013. doi: 10.1590/S1983-14472013000400007

MARTIN-FERNÁNDEZ, J. et al. Variables que condicionan la utilización de la consulta de enfermería en centros de salud de la Comunidad de Madrid. Rev. Esp. Salud Pública, v.87, n.4, p.383392, 2013. doi: 10.4321/S1135-57272013000400008

MARTINS, L.; SILVA, E.; MARQUES, D. Health information in the perspective of Family Health Nurses. REME, v.20, p.e932, 2016.

MATTA, G.C.; MOROSINI, M.V.G. Atenção Primária à Saúde. In: PEREIRA, I.B.; LIMA, J.C.F. Dicionário da educação profissional em saúde. Rio de Janeiro: Fundação Oswaldo Cruz, 2009.

MIRANDA NETO, M. V. et al. Advanced practice nursing: a possibility for Primary Health Care? Rev. Bras. Enferm., v.71, p.716-721, 2018. doi: 10.1590/0034-7167-2017-0672

MUNYEWENDE, P.O.; RISPEL, L.C.; CHIRWA, T. Positive practice environments influence job satisfaction of primary health care clinic nursing managers in two South African provinces. Hum. Res. Health, v.12, n.1, p.1-14, 2014. doi: 10.1186/1478- 
4491-12-27

NICHIATA, L.Y.I. et al. Classificação Internacional das Práticas de Enfermagem em Saúde Coletiva - CIPESC®: instrumento pedagógico de investigação epidemiológica. Rev. Esc. Enferm. USP, v.46, n.3, p.766-771, 2012. doi: 10.1590/S008062342012000300032

OPAS - Organização Pan-Americana da Saúde. Ampliação do papel dos enfermeiros na atenção primária à saúde. Washington: OPAS, 2018.

PATIRAKI, E. et al. Nursing care plans based on NANDA, nursing interventions classification, and nursing outcomes classification: the investigation of the effectiveness of an educational intervention in Greece. Int. J. Nurs. Knowl., v.28, n.2, p.88-93, 2017. doi: 10.1111/2047-3095.12120.

PÉREZ RIVAS, F.J. et al. Effectiveness of nursing process use in primary care. Int. J. Nurs. Knowl., v.27, n.1, p.43-48, 2016. doi: $10.1111 / 2047-3095.12073$

PIMENTA, C.A.M. et al. Guia para construção de protocolos assistenciais de enfermagem. São Paulo: COREN, 2015.

SALVADOR, P.T.C.O.; SANTOS, V.E.P.; DANTAS, C.N. Brazilian dissertations and theses on the interface between nursing process and primary care. REME, v.18, n.2, p.295-302, 2014. doi: $10.5935 / 1415-2762.20140023$

SILVA, C.S. et al. Characterization of the nursing consultation in the care of the person with hypertension and diabetes. Rev. Portal: Saúde Soc., v.2, n.1, p.347-362, 2017.

SILVA, K.M.; SANTOS, S.M.A. The nursing process in family health strategy and the care for the elderly. Texto Contexto Enferm., v.24, n.1, p.105-111, 2015. doi: 0.1590/010407072015000680013. 